

\section{Knowledge That is Not Communicated is Wasted: JOSHA- Open Access with Author Copyright}

\section{by Gerhard G. Steinmann and Roland Mertelsmann}

Abstract:

Universal access to knowledge is a fundamental principle of science and humanities. Today, however, publications of science and humanities are locked behind high paywalls and non-transparent selection procedures. In the efforts to resolve the misery, a first important milestone was the "Berlin Declaration on Open Access to Knowledge in the Sciences and Humanities" of 2003. This year, a second milestone was reached: Eleven European research funding institutions have committed to require from 2020 on that all results from research funded by these institutions are published immediately in compliant Open Access journals or platforms. The negative liabilities of Peer-review procedures, including the "Semmelweis reflex" are shown to represent further barriers for an immediate universal dissemination of knowledge. To overcome inappropriate locking of science and humanities behind walls, the remaining future milestone is that authors retain a non-exclusive copyright.

Key words: Universality, unobstructed access, Berlin declaration, Good Scientific Publication Practice, Semmelweis reflex, unrestricted author copyright 
JOSHA, the Journal of Science, Humanities and Arts, is a multi-disciplinary, multilingual, non-profit open-access electronic journal. Manuscripts undergo Editorial review and rating within 7 days. JOSHA neither pursues an Impact factor nor performs a Peer-review. The authors alone are responsible for the contents of their publication and they hold the copyright, so that they may publish the article elsewhere in the original or a modified version (1).

Today two mayor issues related to the communication of knowledge are currently vehemently discussed. One is the Open Access to Knowledge in science and humanities as a tool to avoid waste of knowledge, the other one the limitations of a Peer-review selection of papers and work to be published.

General and free access to knowledge is a fundamental principle of science and humanities: only work that can be debated, challenged, tested, verified or reproduced by others qualify as scientific work, anything else is wasted. Science and humanities are institutionalized networks of organized criticism and can only function properly if results are made openly available. It is imperative for progress in research, science and humanities, since it builds on recognized results from previous work. Even the international patent laws respect this principle and allow to erect new discoveries on previously established results regardless whether substances or methods are patent-protected or not. Progress in science and humanities can only be optimal if all work and results are made openly available to the public.

But today, as a matter of fact, we have to note that science and humanities are locked behind high paywalls erected by global publishing enterprises (2,3). These paywalls are so incredible and ruthless that even authors of publications have to pay if they want to see their own work.

The current misery is due to the subscription fee-based model of scientific publishing which developed in the history of science, when research papers needed costly mailings, extensive typesetting and photographic reproductions, layout designs, printing, and when hardcopies of journals and reprints needed to be surface- or air-mailed throughout the world. There was no other way to communicate knowledge. As trade in for this service and for about 50 high gloss reprints, authors had no concerns to grant an exclusive copyright to the publisher as part of the conventions.

Since the recent decades, the subscription-based model has been ruled by primarily privatelyowned publishers, who have introduced submission fees for their e-journals in addition to 
subscription fees and gradually increased the cost pressure to scientific libraries and researchers to an agonizing level. In parallel, scientific publishing houses have grown to multibillion concerns. Currently, about five global players lead the scientific publication party, including Reed-Elsevier, Springer Nature, Wiley-Blackwell, Taylor \& Francis and Sage. These five major publishing houses took control of more than $50 \%$ of the publication market and generated an operating margin of about $40 \%$ based on products they simply did not pay for. These companies take a free ride on the back of research, which is funded or sponsored by others, in most cases by public funds, and which is subsequently evaluated by researchers on the basis of voluntary and unsalaried peer review (2).

While moving from print to digital, the publishing process still needs services, but the distribution channels have been completely transformed. There is no valid reason to maintain any kind of double-payed business model for scientific publishing in the digital world. Open Access distribution with authors retaining the copyright is maximizing the impact, visibility, and efficiency of the whole world of science and humanities (3). Open Access publication is definitely one of the most effective measures against the oligarchy of scientific publishers and the disadvantages in efficiency and fairness associated with economic oligopoly.

We find a first important milestone for Open Access in the "Berlin Declaration on Open Access to Knowledge in the Sciences and Humanities", which is an international statement on open access to knowledge. It emerged from a conference on open access hosted by the Max Planck Society in 2003 (4).

\section{Open access contributions must satisfy two conditions:}

"The author(s) and right holder(s) of such contributions grant(s) to all users a free, irrevocable, worldwide, right of access to, and a license to copy, use, distribute, transmit and display the work publicly and to make and distribute derivative works, in any digital medium for any responsible purpose, subject to proper attribution of authorship (community standards, will continue to provide the mechanism for enforcement of proper attribution and responsible use of the published work, as they do now), as well as the right to make small numbers of printed copies for their personal use.

A complete version of the work and all supplemental materials, including a copy of the permission as stated above, in an appropriate standard electronic format is deposited (and thus 
published) in at least one online repository using suitable technical standards (such as the Open Archive definitions) that is supported and maintained by an academic institution, scholarly society, government agency, or other well-established organization that seeks to enable open access, unrestricted distribution, inter-operability, and long-term archiving."

The signature was a celebratory conclusion of a meeting in the Harnack House in Berlin. First to sign included ,die Hochschulrektorenkonferenz, der Wissenschaftsrat, die Deutsche Forschungsgemeinschaft, die Max-Planck-Gesellschaft, die Fraunhofer-Gesellschaft, die Wissenschaftsgemeinschaft Gottfried Wilhelm Leibniz e. V., die Helmholtz-Gemeinschaft Deutscher Forschungszentren und Deutsche Bibliotheksverband“. Up to now, about 600 institutions have signed world-wide (5).

On September $4^{\text {th }}, 2018$, we have welcomed the second milestone: Eleven European research funding institutions from Austria Finland, France, Ireland, Italy, Luxembourg, Netherlands, Norway, Poland, Slovenia, Sweden, UK and the European Research Council have committed to require after January 1st, 2020 that all results from research funded by these institutions are published immediately in compliant Open Access journals or platforms (2). These eleven institutions provide more than half of the European research funding flow, but no German research funding institution has joined the European alliance so far. This is in deep contrast to the famous and enthusiastic Berlin Declaration. Germans obviously have a problem with European initiatives as demonstrated by many other issues, like money laundry, prevention of air pollution prevention, climate protection by $\mathrm{CO}_{2}$ reduction, to name a few.

What are the fears or counter-arguments? Managers of the major publishing houses responded to the commitment of the eleven European institutions with allegations like "undermining the whole research publishing system", "disrupting scholarly communications" and "impinge academic freedom". It is questioned whether the commitment will hold at an constitutional court (2). This ridiculous. We have already a long history of legally compliant publication restrictions in relation to third-party funds. Journalist Thomas Thiel of Frankfurter Allgemeine polemizes about an in his eyes "blind offensive" in favor of the "scientific super powers China and the U.S.A.", who will now receive European scientific publications for free (6). We are concerned how uncritically journalists of serious newspapers pursue the business of large 
publishing houses - No word about the fact that the publishing companies receive their preproducts for free since decades.

It is interesting to note that no scientist has taken a stand against the initiative, only business administrators, lobbyists and journalists.

The "Journal of Science, Humanities and Arts - JOSHA" has been initiated to create an open access to the broad diversity of important discoveries and creativity in the fields of Science, Humanities and Arts. JOSHA also meets a very important requirement of the Berlin declaration, the authors retain the copyright (1). We, the editors of JOSHA, know that Open Access publications cannot be provided for free. The procedures are associated with a certain, but limited amount of cost. This cost, in the case of JOSHA, is covered by two philanthropic institutions, the Academy of Science, Humanities, and Arts (IASHA e. V.) and the BioTheraRoland Mertelsmann Foundation (1).

We, however, are distressed by the demands of the oligarchy. We also do not believe that impact factors and current peer review procedures are essential for an efficient, high-quality publication policy without conflict of interest.

Let us now come to our second point, the Peer-review. It is our conviction that the Holy Grail of the current publication policy "Peer-review" is completely overvalued in the public.

In 2016, three leading European Academies of Sciences, the German Leopoldina, the French Académie des Sciences and the British Society, have agreed on indisputable, but very universal and general guidelines of a Good Scientific Publication Practice. Scientific information should be provided efficiently and at high quality, conflict of interest should be avoided, papers should be checked fairly and selection und compilation should remain in the responsibility of accepted scientists (2).

Peer-review as of today, is, however, a unsalaried burden on the back of voluntary reviewers who are frequently over-worked and often competing in their scientific field. The process is anonym and not transparent. The heterogeneity of peer-reviews reaches from two to three tick marks of an overworked researcher, who is nearly unable to meet the timelines, to major suggestions of new text, interpretations and experiments so that one is inclined to invite the reviewer to co-authorship. 
In contrast, we have learned in the meantime that the most effective control of scientific data is a neutral control of the row data. Traditional Peer-review is unable to execute such a control and by this way to increase data quality in scientific publications. Instead of leaving the assessments to a non-transparent process depending on reviewer personalities, we should look for better methods. The best-established method is currently a systematic on-site "Source Data Verification" by neutral staff. This is expensive and can usually not be conducted by a research institution. However, an option is to upload the row data into open access data repositories or journals, like JOSHA, and to give external scientists the access to look into these data.

Another negative liability of Peer-review is a very human nature and property. Some of you may have heard or read about so-called "Semmelweis reflex"

The Semmelweis reflex (7) is a metaphor for the reflex-like tendency to reject new evidence or new knowledge because it contradicts established norms, beliefs or paradigms. A reflex, or reflex action, is an involuntary and nearly instantaneous movement in response to a stimulus. A reflex is made possible by neural pathways called reflex arcs which can act on an impulse before that impulse reaches the brain. The reflex is then an automatic response to a stimulus that does not receive or need conscious thought.

The term derives from Ignaz Semmelweis, who in 1847 discovered that childbed fever mortality rates fell ten-fold when doctors washed their hands with a chlorine solution between patientsor, most particularly, after an autopsy (at the institution where Semmelweis worked, a university hospital, physicians performed autopsies on every deceased patient). Semmelweis's decision stopped the ongoing contamination of patients - mostly pregnant women — with "cadaverous particles" (8). Unfortunately, Semmelweis published his data not immediately, but with a great delay.

His fellow doctors, however, rejected his hand-washing suggestions, often for non-medical reasons. For instance, some doctors refused to believe that a gentleman's hands could transmit disease. Semmelweis could offer no acceptable scientific explanation for his findings, and some doctors were offended at the suggestion that they should wash their hands and mocked him for it. In 1865, Semmelweis suffered a nervous breakdown and was committed to an asylum, where he died at age 47 of pyemia, after being beaten by the guards, only 14 days after he was committed $(8,9,10)$. 
While there is uncertainty regarding the origin and generally accepted use of the expression, the expression Semmelweis Reflex has been documented and coined by Robert Anton Wilson in his book "The Game of Life" (7).

In the history of science, we have several old and recent examples to demonstrate the reflex. A pronounced one is the reaction of the community of geologists to Alfred Wegener's theory of the continental drift.

Another unpleasant, more recent experience was made by economist George Akerlof. His pioneering article "The Market for Lemons" on the failure of markets in the case of asymmetric information was rejected by the three top journals "American Economic Review", "Journal of Political Economy" and "Review of Economic Studies. Only after three years with hostile Peerreviews, Akerlof was successful with a fourth journal. Years later, Akerlof received the Nobel prize for this work (11).

The Peer-review process is human work and is, as well, susceptible to such effects

JOSHA lists in the meantime an Editorial Board of about 30 members from different disciplines in science and humanities. We are convinced that an Open Access journal with an editorial review by few accepted full-time scientists may effectively communicate honest work as speedily and as broadly as possible.

The current conditions are unsatisfying. The decision of the eleven European research institution is a blessing for all campaigners and pioneers for a free access to results of research that is funded by the public. We congratulate the European research funding institutions for their forward-looking funding policy.

However, a third milestone has to follow: The authors of an article have to retain an unrestricted non-exclusive copyright. Otherwise, scientific Europe will continue to feed the oligarchs of scientific publication. 


\section{References:}

(1) JOSHA - The Journal of the International Academy of Science, Humanities and Arts (IASHA e.V.) - a non-profit association, http://josha-journal.org/en/about

(2) Steinmann GG, Mertelsmann R: Scientific Europe Turns Into a Fair Mode: Open Access Journals Reducing The Cost of Knowledge. DOI: 10.17160/josha.5.7.466

(3) Schlitz M: Science Without Publication Paywalls a Preamble to: cOAlition S for the Realisation of Full and Immediate Open Access. https://www.scienceeurope.org/wpcontent/uploads/2018/09/cOAlitionS_Preamble.pdf

(4) https://openaccess.mpg.de/Berlin-Declaration

(5) http://openaccess.mpg.de/3883/signatories

(6) Thiel T: Blinde Offensive. Frankfurter Allgemeine, 14.9.2018

(7) Wilson RA: The Game of Life, New Falcon Publications. 1991

(8) https://en.wikipedia.org/wiki/Semmelweis_reflex

(9) Levitt SD: "4". Super Freakanomics. William Morrow. 2009

(10) https://en.wikipedia.org/wiki/Ignaz_Semmelweis

(11) Plickert P: Die Tyrannei der Top-Journale. Frankfurter Allgemeine Sonntagszeitung, 21.10.2018; with reference to Gans JS, Shepherd GB: How Are the Mighty Fallen: Rejected Classis Articles by Leading Economists. Journal of Economic Perspectives. 1994 